\title{
The effect of harness suspension on a simulated maintenance task efficacy in the renewable energy industry.
}

STEWART, A.D., GARDINER, M., MACDONALD, J. and WILLIAMS, H. 


\title{
The effect of harness suspension on a simulated maintenance task efficacy in the renewable energy industry
}

\author{
Arthur D. Stewart*, Matthew Gardiner, Jonathan MacDonald \& Hector Williams. \\ School of Health Sciences, Robert Gordon University, Ishbel Gordon Building, \\ Garthdee Road, Aberdeen AB10 7QG, UNITED KINGDOM.
}

*Corresponding author

\section{Abstract}

Building, bridge or wind turbine maintenance requires manual dexterity tasks by a specialist rope-access trained workforce via two principal means: harness suspension of individual workers from above, or deployment of a suspended platform or cradle from which workers access the structure to be maintained. Currently no published research compares accuracy and efficiency of simulated maintenance tasks between these modalities. This study investigated manual dexterity task performance of peg placement and shape delineation in seated, standing and suspended environments in 16 healthy controls and 26 professional rope-access trained individuals. Both seated and standing assessments were superior to those suspended, and height of suspension, total mass and years of experience had no influence on the task outcome. These findings suggest that, where feasible, cradle suspension mechanisms which permit standing maintenance are favourable in terms of task efficacy and where feasible, should be considered for deployment in wind energy and other engineering applications.

Key Words

Rope-access, harness suspension, task performance, pegboard, shape delineation

Highlights

- A novel simulated maintenance task was developed for rope-access maintenance workers

- Performance was compared standing versus suspended from two heights

- Task accuracy and efficiency were detrimentally affected by harness suspension

- Height of suspension, years of experience and body mass had no effect

\section{Introduction}

Global energy production is increasingly relying on renewable sources, in particular onshore and offshore wind technologies. In a burgeoning sector, wind turbines have proliferated since their introduction in the early 1990s and typically have a lifespan of about 25 years (Kolios \& Martínez Luengo, 2016). After their installation, these turbines require regular scheduled maintenance in order to maintain efficient power 
generation capability. For example, mechanical maintenance may require inspection, adjustment and replacement of bearings and gearing mechanism housed in the nacelle housing beside the hub. In addition, wind turbines must be maintained to protect against environmental degradation. Erosion of the exposed surfaces of the structure result from natural processes such as ultraviolet light, ice, lighting strike, and especially micro-pitting on surfaces result from water contact (Offshore Renewables Energy Catapult, 2018). In particular, this is an issue for the turbine blades themselves which experience surface degradation, predominantly in the region of tip where the velocity of impact with water droplets is greatest. The resulting surface degradation substantially reduces blade efficiency in generating power and occasionally results in blade failure and breakage which significantly increases operational expenditure. While regular planned maintenance can maximise the chances of a turbine fulfilling its life expectancy, there is a buoyant market for retro-fitting components have the potential to extend the working life well beyond this timespan (Wind Prospect, 2018).

Blade maintenance of offshore wind turbines typifies many harness suspension tasks. This involves inspecting the entirety of a wind turbine blade surface in situ. Damaged areas (occurring as a result of factors such as water erosion, lightning, ultraviolet degradation and algal growth) are delineated and subsequently systematically ground, filled and cured to preserve a smooth and efficient shape. This work is traditionally carried out by specialist rope-access technicians in a process which becomes increasingly necessary as a turbine ages. The Industrial Rope Access Trade Association (IRATA) defines 'rope access' as "a form of work positioning where the person is suspended by means of personal fall Prevention equipment (rope, harness etc.) used in tension". This secure suspension in tension defines what is referred to as 'harness suspension' in this study which included, like industrial contexts, a secondary safety system connected to the individual.

Like similar rope access applications, blade maintenance can be undertaken via two principal means - by harness suspension from an anchor point above or via a cradle and habitat mechanism on which the engineer stands, which accesses the blade. The harness suspension method using individual rope access engineers benefits from more rapid deployment and egress in the event of adverse weather conditions. Its principal disadvantages centre on the potential threat to circulation, orthostatic syncope (Adisesh et al., 2011) and consequent suspension trauma, which is potentially fatal (Lee \& Porter, 2007). While modern safety harnesses and protocols have lowered the risk of these to minimal levels, they may not have been eliminated entirely, although the poorer upper extremity musculoskeletal health of the maintenance engineers relative to controls may be imposed by work tasks and not the access technique involving full body harness suspension (Vikne et al., 2015).

Harness-suspended engineers must also overcome the challenge of generating purchase when using tools against a vertical surface. To avoid reactive forces pushing them away from the blade surface they require stabilisation via tethering and incur additional muscular effort when exerting high forces. By contrast, a cradle and habitat approach, is safer for individuals in terms of health, but is considerably more of an 
engineering challenge to achieve in practice. Cradle devices large enough to encapsulate the blade cross section must be deployed by boat and winched up and down from the hub or the turbine tower. Depending on anchoring mechanisms, these take considerable time to install, but have the advantage of offering the maintenance engineers a walking platform which presents more favourable for body configuration than harness suspension, and also a degree of weather protection for conducting the work.

Fine motor tasks are undertaken across many industries. Since the pioneering work by Fitts (1954) identified a movement can be subdivided into rapid gross and slower fine components, a multitude of studies have considered the interplay of posture and task accuracy. For example, Berrigan et al. (2006) demonstrated longer movement duration in an aiming task with smaller target, and differences between task performance and standing and seated for a small target, noting a complex interplay of posture, movement, speed, and accuracy. For more gross, heavier object movement, the stabilisation of the body and degrees of freedom are perhaps more influential. The use of a grinder has been estimated to equate to $60 \pm 40 \mathrm{~N}$ for up to $3.0 \pm 1.5$ hours per day maximum (Lindquist \& Skogsberg, 2007). When the operator is standing on a cradle system, the force of bodyweight and friction-coupling stabilises this horizontal force when working on the blade surface. When suspended, the energetic cost of bracing needs to be considered, along with the injury risk of continuous work for extended periods.

While both harness-suspension and cradle approaches are in widespread use, across various applications, there is currently no research evidence to assess task efficacy might be favoured by either approach. As a result, this study sought to determine task efficacy and work quality in laboratory and field tests of manual dexterity which replicate fine motor tasks routinely undertaken by wind energy technicians, firstly in controls to develop the protocols and to estimate effect size and subsequently in rope-access professionals.

\section{Methods}

\subsection{Population and sample}

The study recruited a total of 42 individuals in two separate samples. Experiment one used a convenience sample of 9 male and 7 female adults who were students and staff of Robert Gordon University with no previous experience of harness suspension and was conducted in the scanning and ergonomics laboratory on campus.

Experiment two was based in an industrial hangar and recruited 25 male and 1 female qualified rope-access technicians with a minimum of one year's professional experience from rope access training providers in Aberdeen and Kinross, UK. All provided informed written consent and the study was approved by the school research ethics committee (study SHS/18/23). Characteristics of the participants are provided in tables $1 \mathrm{a}$ and $1 \mathrm{~b}$, and manual dexterity scores for controls and ropeaccess technicians appear in tables 2 and 3 respectively. 
There was an absence of comparable studies to base a sample size estimation from. However, a related study of arm support in seated and standing used 11 participants (Odell et al., 2007), while a reaction time study in upright and twisted postures used 21 participants (Newell \& Mansfield, 2008). Both these had the body supported either in standing or sitting, straight or twisted postures. Participants of the present study were required to be of working age (18-66 y) and provided written consent to take part. It was hypothesised that with a control sample, an indication of the effect size of tripod suspension might be possible, although with the likelihood that rope access engineers would be better than controls at balance and task efficacy, it was hypothesised that the effect would be smaller in rope access technicians. Each was screened for physical activity readiness using the PAR-Q (2002 version, based on original research validation by Thomas et al., 1992) answering all questions in the negative, and were required to be free from musculoskeletal injury for 6 months and to be apparently healthy at the time of testing. Participants were screened for contrast sensitivity using a Pelli-Robson contrast sensitivity chart at $100 \mathrm{~cm}$, (Haag Streit, UK) to include only those participants who have adequate visual ability to detect a small difference in contrast.

Stature and mass were assessed using a Seca portable stadiometer (model 217) and Seca digital scales (model 875; Seca, Frankfurt, Germany) using standard protocols (Stewart et al., 2011). Controls were measured wearing indoor clothing and without shoes. Rope access technicians (who arrived for the study directly from bridge maintenance work nearby) wore personal protective equipment and protective work boots, but removed harnesses and helmets for these measures. The additional mass of PPE and boots ( $3.2 \mathrm{~kg}$ ) and height advantage offered by industry work boots (3.6 $\mathrm{cm}$ ) were subtracted accordingly.

Participants underwent familiarisation (equivalent to two testing cycles) prior to the testing session, to ensure habituation and learning of the required tests and, additionally for controls, familiarisation with the harness suspension system.

\subsection{Test Conditions}

Experiment one involved healthy adult volunteers with no rope access training or experience. Tests were performed both seated at a horizontal bench surface and under harness suspension conditions using a portable industrial lifting tripod (Didsbury Lightweight tripod, Didsbury, UK). For experiment two, tests were performed standing, suspended under the same tripod, and additionally, with a $7.5 \mathrm{~m}$ suspension from the apex of the testing hangar, at an equivalent height above the floor as depicted in figure 1. All participants performed the tests under their respective conditions in a randomised order (www.randomize.org) to mitigate any residual learning effect of the tests. 


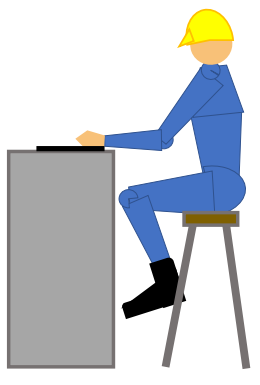

Seated

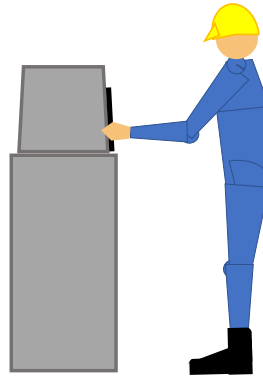

Standing

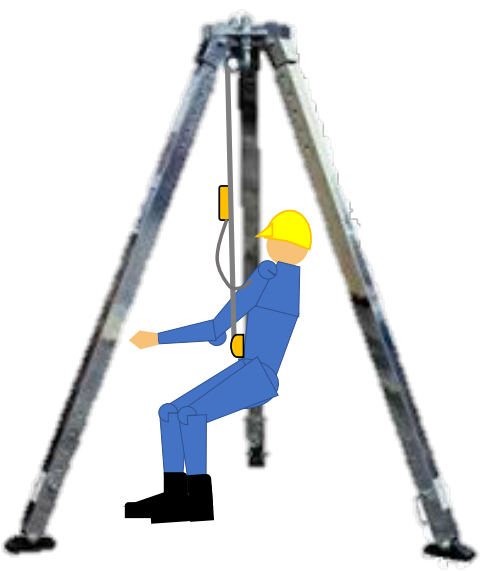

Tripod suspended $(2.2 \mathrm{~m})$

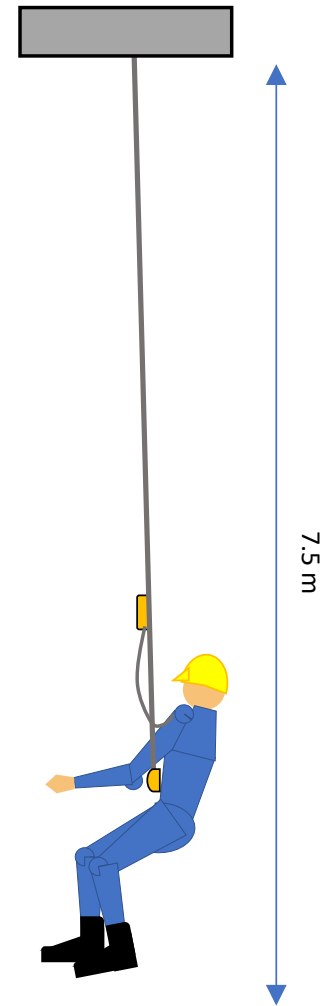

Hanging from $7.5 \mathrm{~m}$

Figure 1. Schematic showing the three conditions for the tests (not to scale): Controls underwent seated and tripod suspended conditions; rope access personnel underwent standing, tripod suspended and hanging conditions.

\subsection{Dexterity Tests}

Selection of suitable tests required them to be standardised and portable for field use, and to yielding data relatively quickly. Tests of manual dexterity were conducted using the preferred hand only and involved a modified peg placement task and a computerised shape delineation task. A modified Purdue pegboard test (Lafayette Industries, USA) was conducted involving individually grasping and placing 10 pegs, washers and collars in sequence as quickly as possible (depicted in Figure $3 \mathrm{~L}$ ). Participants for both experiments were given two familiarisation trials in a seated position. For the test itself, in experiment one the pegboard was oriented horizontally on the lab bench, with a $10 \mathrm{~cm}$ gap between the seated participant's torso and the edge of the bench. For tripod suspension, delineated with floor markings to locate the rolling furniture standardised the same relative position. In experiment two, the pegboard was secured in a vertical orientation $35 \mathrm{~cm}$ in front of the standing participants. Harness suspension for both tripod and $7.5 \mathrm{~m}$ hanging was carefully controlled to an equivalent horizontal distance within the reach envelope. (depicted 
in figure 2). This distance was not adjusted according a person's anthropometric size, therefore it is accepted that taller people had a 'less stretched' reach than shorter individuals.

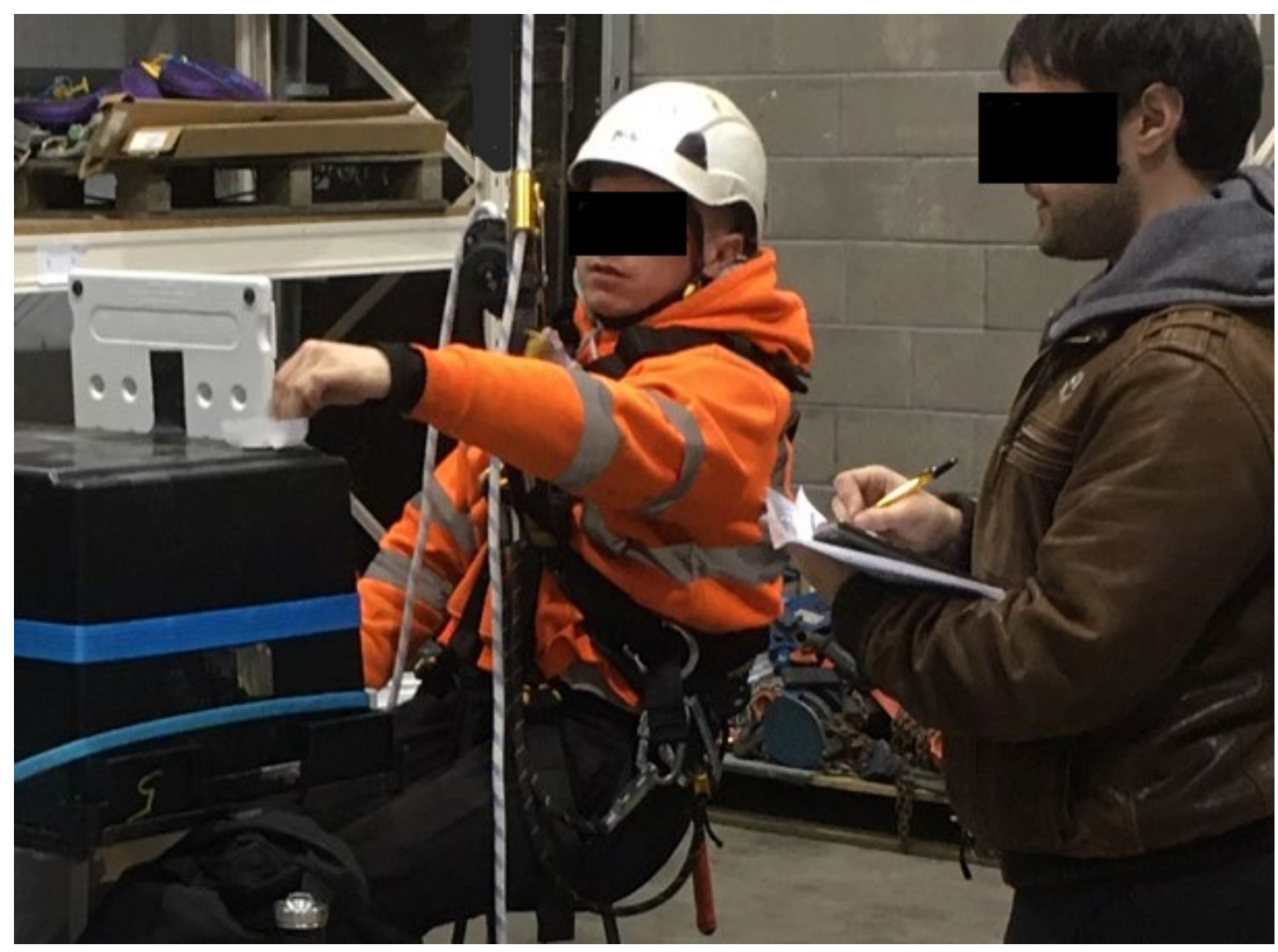

Figure 2. Testing of rope access technicians at SPAN Access Solutions, Kinross, UK.

A visual inspection test "TIMDEX" was specifically designed to simulate the precise identification of micro-pitting on a blade surface (Stewart et al., 2019). This involved a selection of low contrast shapes relative to the background being traced on a touchscreen laptop using the MS PowerPoint drawing tool (Figure 3, R). After familiarisation with 8 shapes, participants were given test shapes (4 in experiment one and 2 in experiment two). According to the speed-accuracy trade off on motor performance elucidated by Fitts (1954), it was decided to allow participants up to $15 \mathrm{~s}$ per shape to delineate them with the index finger of the preferred hand. Completed shapes were flood-filled, saved as PNG files and processed using the 'magnetic lasso' feature of Adobe Photoshop (version CC, 2017) and compared with the original template for precise pixel area using ImageJ $1.51 \mathrm{r}$ software (National Institutes of Health, USA). The total area of excursions beyond the shape boundary or incursions inside the shape boundary were summarised in a global error score calculated as the square root of the sums of the squares of the delineated-actual area differences, expressed as a percentage. Verbal feedback on shape delineation performance was provided to participants after their familiarisation session. Standardised positioning was adopted for this test, as for the pegboard test. 


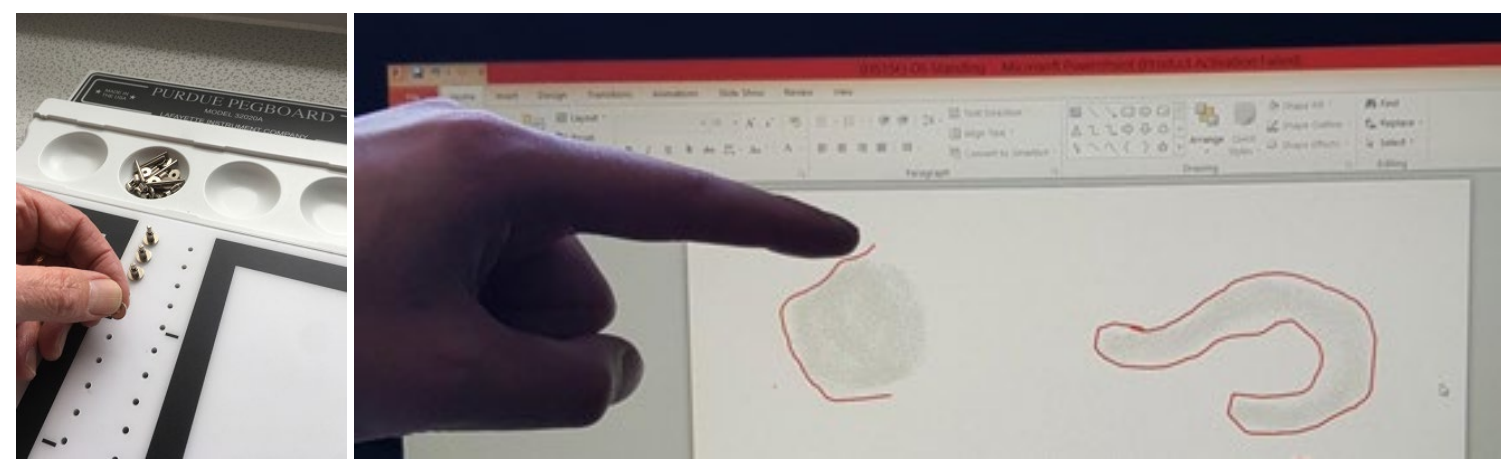

Figure 3. L: Modified Purdue pegboard test; R: TIMDEX on-screen test

\subsection{Statistical methods}

The condition state of tripod suspension or roof suspension was compared against the baseline states of sitting (experiment 1 ) and standing (experiment 2) with paired samples t-tests, and Cohen's d estimate of effect size. A general linear model with repeated measures using body mass and years of experience as covariates was constructed for each test of rope access technicians for both tests with Bonferroni's adjustment for multiple comparisons. The level of significance was set at $P<0.05$. Precision of analysis for the TIMDEX test was calculated for intra-tester and intertester technical error of measurement in pixel area in a sample of 8 individuals and expressed as a percentage of the mean value.

\section{Results}

Physical characteristics of the participants are illustrated in table 1.

\begin{tabular}{lccc} 
Controls & Male $(\mathrm{n}=9)$ & Females $(\mathrm{n}=7)$ & Combined $(\mathrm{n}=16)$ \\
\hline Age $(\mathrm{y})$ & $25.4 \pm 4.6$ & $30.1 \pm 9.4$ & $27.0 \pm 7.3$ \\
Stature $(\mathrm{cm})$ & $175.9 \pm 8.2$ & $163.9 \pm 2.5$ & $171.2 \pm 8.8$ \\
Mass $(\mathrm{kg})$ & $85.4 \pm 17.3$ & $61.1 \pm 6.6$ & $74.8 \pm 17.7$ \\
\hline & & & \\
Rope-access Technicians & Male $(\mathrm{n}=25)$ & Females $(\mathrm{n}=1)$ & Combined $(\mathrm{n}=26)$ \\
\hline Age $(\mathrm{y})$ & $32.4 \pm 8.6$ & 42.0 & $32.8 \pm 8.6$ \\
Stature $(\mathrm{cm})$ & $178.5 \pm 6.6$ & 177.8 & $178.3 \pm 6.5$ \\
Mass $(\mathrm{kg})$ & $85.0 \pm 16.5$ & 70.3 & $84.4 \pm 16.4$ \\
Years of experience & $5.7 \pm 5.9$ & 8.0 & $5.8 \pm 5.8$ \\
\hline
\end{tabular}

Table 1. Physical characteristics of participants

Precision of shape analysis across eight individuals' shape data varied between 0.1 and $0.9 \%$ within each tester, and was $0.6 \%$ for inter-tester analysis. 


\section{Experiment One (controls).}

Results for the Pegboard and TIMDEX tests are in table 2.

\begin{tabular}{ccccc}
$\mathrm{n}=16$ & seated & tripod & $\mathrm{P}$ & $\mathrm{d}$ \\
\hline Pegboard test $(\mathrm{s})$ & $77.3 \pm 12.9$ & $89.1 \pm 19.3$ & $<0.001$ & 0.68 \\
TIMDEX error $(\%)$ & $38.6 \pm 15.1$ & $51.7 \pm 12.2$ & 0.005 & 0.87 \\
\hline
\end{tabular}

Table 2. Manual dexterity scores for in control participants seated and with harness suspension (mean and SD; Cohen's d effect size)

\section{Experiment Two (rope access technicians)}

The analysis of variance revealed that there were differences according to participant state (standing $\vee$ tripod suspension $\vee 7.5 \mathrm{~m}$ suspension) for the pegboard test $(P<0.0001)$ as indicated in figure 4 , and shape delineation $(P=0.018)$ as in figure 5 , however for shape delineation, the pairwise comparison using Bonferroni adjustment yielded no significance between standing and tripod ( $P=0.153)$ and borderline significance between standing and hanging $(P=0.054)$. Neither total body mass nor years of experience had an interaction with task performance for either test $((P>0.05)$.

Results for the comparison of tripod and hanging suspension with standing are given in table 3.

\begin{tabular}{lccccccc} 
& standing & tripod & $\mathrm{P}$ & $\mathrm{d}$ & hanging & $\mathrm{P}$ & $\mathrm{d}$ \\
\hline Pegboard test & $105.8 \pm$ & $132.6 \pm$ & $<0.001$ & 0.93 & $137.5 \pm$ & $<0.001$ & 0.99 \\
(s) $\mathrm{n}=26$ & 21.6 & 29.4 & & & 33.2 & & \\
& & & & & & & \\
TIMDEX error & $25.1 \pm$ & $30.3 \pm$ & 0.153 & 0.39 & $34.1 \pm$ & 0.054 & 0.55 \\
(\%) $n=26$ & 11.8 & 15.0 & & & 19.4 & & \\
\hline
\end{tabular}

Comparison of tripod and hanging with standing condition (after Bonferroni adjustment). Hanging was not significantly different from tripod suspension for the pegboard test $(P=$ 0.907) or the shape delineation ( $P=0.661)$.

Table 3. Manual dexterity scores for rope access technicians standing, harness suspended from tripod, and hanging from $7.5 \mathrm{~m}$ (mean $\pm \mathrm{SD}$; Cohen's d effect size). 


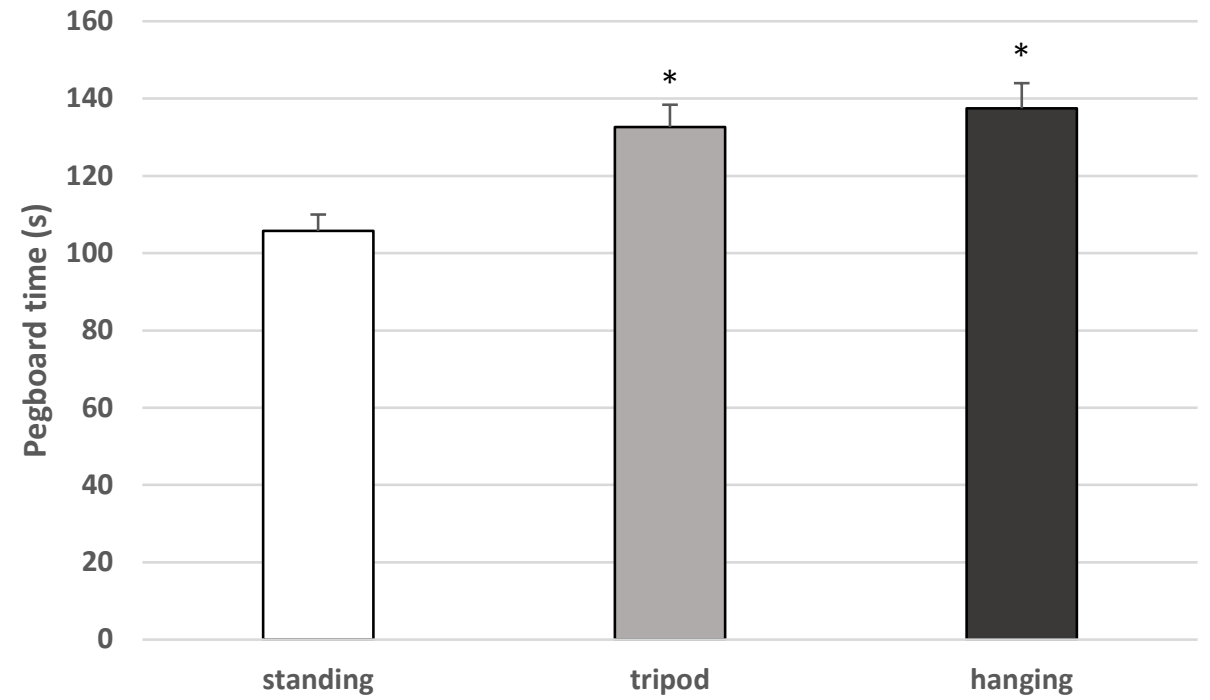

Error bars represent 1SE * different from standing $\mathrm{P}<0.001$

Figure 4. Pegboard time under different suspension conditions

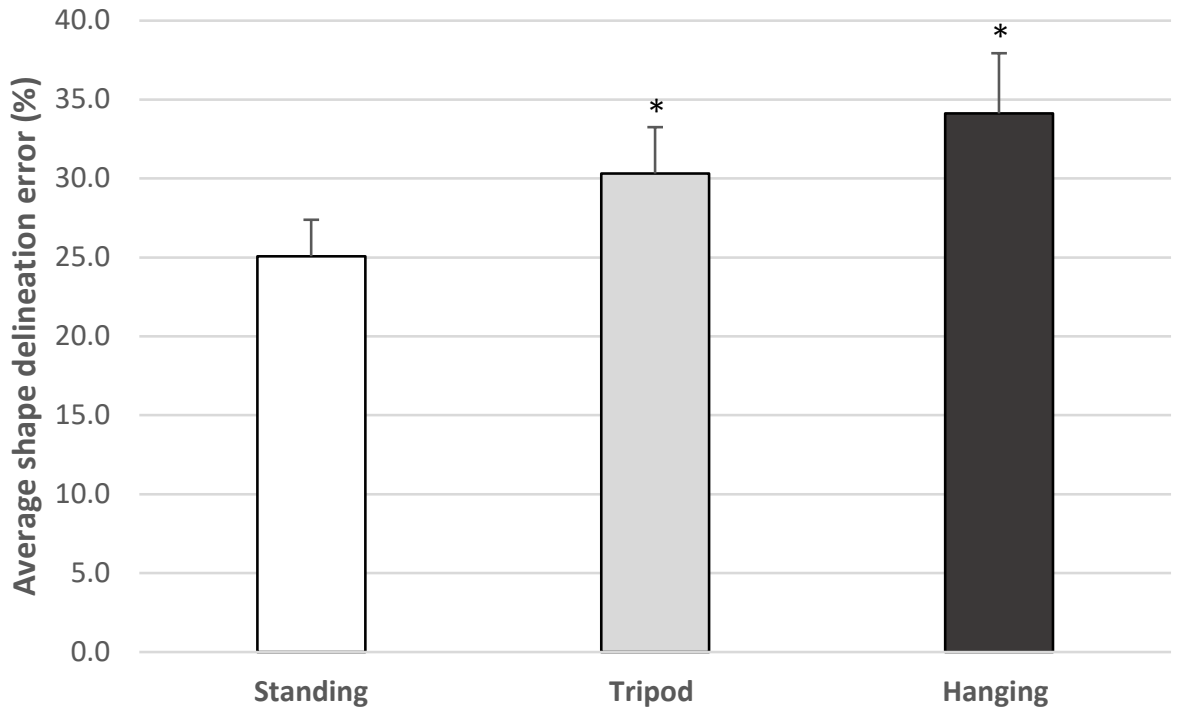

Error bars represent 1SE; * different from standing $\mathrm{P}<0.001$

Figure 5. Shape delineation error under different suspension conditions

\section{Discussion}

\subsection{Key Findings}

Seated or standing is superior to harness suspension in terms of task efficiency based on pegboard time, both for untrained and experienced rope access technicians. While the shape delineation data are less clear-cut, they also display a similar effect. There does not appear to be a difference between long versus short suspension length in terms of these findings amongst rope access technicians. Rope-access years of experience failed to enhance task performance while suspended, evidenced by no interaction with standing - suspended difference for either test. Although this was 
surprising and somewhat counter-intuitive, clearly other factors were more influential, suggesting that after their training to become qualified rope access technicians, years of experience had no additional effect. It is likely that experience might result in other benefits such as optimising comfort during the task, or being able to continue working for longer without discomfort, neither of which was measured in this study.

The study design precluded direct comparison between controls and technicians. It could be hypothesised that experienced rope access technicians' superior stability relative to controls in the tripod suspension would carry over to manual dexterity task performance, however the results from the present study do not bear this out.

While the literature has no examples of fine motor skills amongst this occupational group, there is mixed evidence among other professions. Experienced podiatrists outperformed novices in strength and speed of fine motor tasks, novices were superior at coordination and sensory ability (Causby et al., 2018). Musicians outperformed non-musicians in timing but not spatial aspects of finger placement (Kincaid et al., 2002). As a result, it is not certain whether advantages by professionals over controls may be negated by other factors. In the present study, protocol differences did not facilitate direct comparison between groups or environments. The baseline measurement for controls was seated at the lab bench, while for rope access technicians, it was standing. Standing work allows the operator greater mobility and facilitates greater force generation (Lindquist \& Skogsberg, 2007) while sitting constrains extraneous movement and stabilises dexterity movements relative to standing, avoiding postural sway and balance issues. Controls performed the pegboard tests on a horizontal surface while rope-access technicians performed the test when the board was constrained in a vertical position, upon advice from the industry to make the test more context-specific. A horizontal pegboard orientation is advantageous on two counts: for forearm support during aiming and force generation in pressing pegs into the slots, and also by gravity assisting the threading of collars and washers on the pegs. In addition, when harness suspended, a vertical pegboard orientation generates unwanted as pegs are placed onto it, as a result of equal and opposite reactive forces. In addition, controls were measured in a laboratory whose temperature remained very close to $18^{\circ} \mathrm{C}$ throughout the measurement acquisition. By contrast, rope access workers were measured when they were available in November and December, and not committed to the bridge repair work which occupies them throughout the warmer months. It is well-recognised that manual dexterity is detrimentally affected by cold temperatures, and despite being habituated to site work for 10 months of the year the ambient temperatures in the hangar for rope access workers was between 6 and $10^{\circ} \mathrm{C}$. Because the test of manual dexterity required hands and fingers to manipulate the objects, gloves could not be worn. Previous evidence summarised in Heus et al. (1995) illustrated that decrements in functional dexterity have been detectable for finger skin temperatures below $20^{\circ} \mathrm{C}$ but important losses in dexterity occur at hand skin temperature of $15^{\circ} \mathrm{C}$ or below. Earlier work on task performance and cooling showed decrements in pegboard assessments and knot tying in military personnel (Lockhart et al. 1975). While this 
temperature difference limits the comparability between controls and rope access technicians, randomisation of the order participants are effectively their own controls for assessing the effect of suspension on dexterity. Lastly, it is possible to speculate that finger size may have been greater amongst the technicians due to a predominance of manual work. Although not measured, if this were the case, it could have detrimentally affected both tasks.

Multiple joints (degrees of freedom) are implicated in manual tasks undertaken in occupational work, and control of unwanted movement involves stabilisation via higher nervous system commands, with the result that controlled and uncontrolled degrees of freedom may be described and tested (Scholz \& Schöner, 1999). In-built redundancy into the human body's motor control system involve synergistic actions of motor effectors (neural organisations and joints) which increase with task complexity or uncertainty to facilitate successful task completion (Kim et al., 2012). The need for maintaining wind turbine blades is important from several standpoints. At a strategic level there is a cost function assigned to blade repair which is directly related to the levelised cost of energy (LCOE) due to efficiency gains following repairs (Offshore Renewables Energy Catapult, 2018). At a human level this is achieved via highly skilled manual work which commands musculoskeletal effort and its associated risk on maintenance personnel. Evidence from the present study suggests that work of this type is likely to be better accomplished by standing rather than being suspended, both in terms of accuracy (from the shape delineation test) and in terms of efficiency (from the pegboard test). However, there may be a third aspect to the advantage offered by standing on a platform which relates to musculoskeletal risk. Because harness suspended workers must tense themselves when exerting a force on the blade surface (required for grinding out pitting and other damage) this requires an antagonistic force which they must either exert individually or with a partner. Such isometric effort also has a cost, and in related work, occupational outcomes such as fatigue and pain may result from decreased motor variability of arm movements (Srinivasan et al., 2015). Elsewhere, evidence suggests task performance may be prioritised over energy expenditure for a contact-based task (Balasubramanian et al., 2009).

\subsection{Implications for Practice and future work}

Implications of these findings suggest that standing tasks are likely to produce faster and more accurate work than with harness suspension. In practical terms, there are many other considerations as to which approach should be deployed, according to the precise nature of the task, weather variability and other environmental or logistical factors. While typical blade maintenance tasks might involve a predominance of work which has a more gross motor function than the finer dexterity assessed in the present study, in practice this would have been very difficult to measure experimentally without increased cost and risk. Nevertheless, until such future work is conducted, it would seem reasonable to assume that the arguments favour standing versus suspended work will apply. 
These factors and the industry's regulatory framework will become increasingly important within the rapidly developing but relatively young sector. Because industrial safety is paramount, it is also necessary to consider the demographics of the occupational group and how this may be anticipated to change. It is possible to draw parallels with the offshore oil \& gas industry which underwent profound demographic change over a generation between 1984 and 2014 (Stewart et al., 2017), with median age of employees increasing by 9 y. It would seem prudent to anticipate that the offshore wind energy industry could expect a similar demographic trajectory and that an age increase of this magnitude might conceivably affect functional performance. While manual dexterity might be affected less than other factors such as ladder ascent, peer and self-rescue, and emergency response, further research is required to ascertain this. Ultimately, these study data, together with supporting evidence elsewhere suggest the favourability of cradle systems in terms of work efficacy as well as minimising injury risk, although it is likely that harness suspension approaches will still be necessary under some circumstances.

\section{Declaration of competing interest}

The authors declare the following financial interests/personal relationships which may be considered as potential competing interests: This study was supported by the Blade Access System Environment study, funded by Innovate UK grant no. 104214 (BASE)/IFS2079 and a consortium led by SPAN Access Solutions Ltd, Kinross, UK.

\section{Acknowledgement}

This study was supported by the Blade Access System Environment study, funded by Innovate UK and a consortium led by SPAN Access Solutions Ltd, Kinross, UK, including Turner Iceni Ltd, Turner Access Ltd, Robert Gordon University, University of Dundee, and Offshore Renewable Energy Catapult Ltd. A. Stewart designed the study and authored the paper; J. MacDonald and M. Gardiner undertook practical data collection both in Aberdeen and Kinross, and H. Williams undertook statistical analysis.

\section{References}

Adisesh, A., Lee, C. \& Porter, K. (2011). Harness suspension and first aid management: development of a guideline. Emergency Medicine Journal, 28, 265268. doi:10.1136/emj.2010.097246

Balasubramanian, R., Howe, R.D. \& Matsuoka, Y. (2009). Task performance is prioritised over energy reduction. IEE Transactions on Biomedical Engineering, 56, 1310-1317.

Berrigan, F., Simoneau, M., Martin, O., \& Teasdale, N. (2006). Coordination between posture and movement: interaction between postural and accuracy constraints. Experimental Brain Research, 170, 255-264. 
Causby, R., McDonnell, M., Reed, L. \& Miller, S. (2018). Measuring dexterity in the podiatrist population: a cross-sectional comparison of novice students and experienced podiatrists. BMC Medical Education, 18:181 doi: 10.1186/s12909-018-1276-1.

Fitts, P. M. (1954). The information capacity of the human motor system in controlling the amplitude of movement. Journal of Experimental Psychology, 47, 381-391.

Heus, R., Daanen, H. \& Havenith, G. (1995). Physiological criteria for functioning of hands in the cold. Applied Ergonomics, 26, 5-13.

Kim, M.J., Karol, S., Park, J., Auyang, A., Kim, Y.H., Kim, S. \& Shim, J.K. (2012). Interjoint synergies increase with motor task uncertainty in a whole-body pointing task. Neuroscience Letters, 512, 114-117.

Kincaid, A.E., Duncan, S. \& Scott, S.A. (2002). Assessment of fine motor skill in musicians and nonmusicians: differences in timing versus sequence accuracy in a bimanual fingering task. Perceptual and Motor Skills, 95, 245-257.

Kolios \& Martinez-Luengo (2016) The end of the line for wind turbines. Renewable Energy Focus http://www.renewableenergyfocus.com visited 20/005/18.

Lee, C. \& Porter, K.M. (2007). Suspension Trauma. Emergency Medicine Journal, 24, 237-238. Doi: 10.1136/emj.2007.046391.

Lindquist, B. \& Skogsberg, L. (2007). Power Tool Ergonomics: Evaluation of Power Tools. Atlas Copco. ISBN: 978-91-631-9900-4. p 22.

Lockhart, J.M., Kiess, H.O. \& Clegg, T.J. (1975). Effect of rate and level of lowered finger surface temperature on manual performance. Journal of Applied Physiology 60, 106-113.

Newell, G.S. \& Mansfield, N.J. (2008). Evaluation of reaction time performance and subjective workload during whole body vibration exposure while seated in upright and twisted postures with and without armrests. International Journal of Industrial Ergonomics, 32, 499-508.

Odell, D., Barr, A., Goldberg, R., Chung, J. \& Rempel, D. (2007). Evaluation of a dynamic arm support for seated and standing tasks: a laboratory study of electromyography and subjective feedback. Ergonomics, 50, 520-535.

Offshore Renewables Energy Catapult (2018). Personal Communication.

Scholz, J.P. \& Schöner, G. (1999). The uncontrolled manifold concept: identifying control variables for a functional task. Experimental Brain Research, 126, 289306.

Srinivasan, D., Mathiassen, S.E. Samani, A. \& Madeleine, P. (2015). The combined influence of task accuracy and pace on motor variability in a standardised repetitive precision task. Ergonomics, 58, 1388-1397.

Stewart, A., Marfell-Jones, M., Olds, T. \& de Ridder, H. (2011). International Standards for Anthropometric Assessment. International Society for the Advancement of Kinanthropometry, Lower Hutt, New Zealand.

Stewart, A., Gardiner, M., MacDonald J. \& Hume, P. (2019). Touchscreen Inspection and Manual Dexterity (TIMDEX) task. J.E. Lindsay Carter Kinanthropometry Archive. http://jelckca-bodycomp.com

Stewart, A., Ledingham, R., Furnace, G. \& Williams, H. Nevill, A. (2017). Shape change and obesity prevalence among male UK offshore workers after 30 years: new insight from a 3D scanning study. American Journal of Human Biology. 00:e22992 doi.org/10.1002/ajhb.22992 
Thomas S., Reading, J. \& Shephard, R. (1992). Revision of the Physical Activity Readiness Questionnaire (PAR-Q). Canadian Journal of Sport Sciences, 17, 338345.

Vikne, H., Jebens, E., Elka, S., Knardahl, S. \& Veiersted, K.B. (2015). Working suspended in a harness rig: A comparative study of musculoskeletal health complaints in rope access technicians and controls. Work, 56, 291-300.

Wind Prospect (2018) https://www.windprospect.com.au/technologies?t=offshore visited 29/05/2018. 